\title{
Children's Understanding of Psychogenic Bodily Reactions
}

\author{
Paul C. Notaro, Susan A. Gelman, and Marc A. Zimmerman
}

\begin{abstract}
One hundred twenty-eight children in preschool through fifth grade (range $=4,3-11,4)$ and 76 adults serving as a comparison group participated in two studies that examined how children reason about psychogenic bodily reactions, that is, ailments or nonconscious physiological responses with origins in the mind (e.g., stress-induced headache). Psychogenic bodily reactions provide an opportunity to study how children integrate knowledge between the domains of bodily response and psychology. In Study 1, participants were asked whether various familiar psychogenic bodily reactions were possible (e.g., can someone get a tummyache from worrying?). In Study 2, participants were presented with a novel domain (hypothetical "aliens" from outer space) and were asked whether various unfamiliar bodily conditions (e.g., toes swelling) could arise from various physical or psychological causes. As predicted, adults typically reported that psychogenic bodily reactions were possible, and that unfamiliar bodily conditions could result from either psychological or physical causes. In contrast, young children typically denied that psychogenic bodily reactions could occur and predicted that unfamiliar bodily conditions resulted from physical causes only. The results support a developmental path: younger children view psychogenic bodily responses as wholly physical, but with age, view them as both physical and psychological phenomena.
\end{abstract}

\section{INTRODUCTION}

Researchers have shown strong interest in children's developing "theories" about the world (Carey, 1985; Gopnik \& Wellman, 1994; Wellman \& Gelman, 1992). A primary assumption of this work is that theories are domain specific: Children construct separate theories for physics, psychology, and perhaps biology, each with their own ontological commitments, causal processes, and organization (Carey, 1985; Gopnik \& Wellman, 1994). To the extent that children do possess domain-specific theories, they should honor firm distinctions between domains. And indeed, a wealth of evidence suggests that young children honor an ontological distinction between mental/ psychological phenomena and bodily/physical phenomena (Coley, 1995; Estes, Wellman, \& Woolley, 1989; Harris, Brown, Marriot, Whithall, \& Harmer, 1991; Hatano \& Inagaki, 1994; Wellman \& Estes, 1986). Few investigators, however, have studied how children understand concepts that simultaneously involve two domains.

The focus of this article is on how children reason about phenomena that cross ontological domains. The study of domain interaction is necessary to gain a complete picture of children's basic cognitive processes. Although cross-domain reasoning is perhaps not as common as within-domain reasoning, it does occur. Psychogenic bodily reactions (bodily ailments with origins in the mind) may be one of the most frequent, and include such discomforts as tension headaches or stress-induced rashes. In fact, pediatricians report that $17 \%$ to $50 \%$ of their patients experience psychogenic reactions (Garralda \& Bailey, 1987, 1990; Kaplan, 1980; Reister, Tress, Schepank, \& Manz, 1989).
Other examples of cross-domain phenomena include biofeedback (in which mental states have physical, bodily effects) and mind-altering drugs (in which bodily states have psychological effects).

The present studies examined children's understanding of psychogenic bodily reactions. A psychogenic reaction has a psychological cause but a physical outcome. Thus, to understand what is happening to the body when one experiences a psychogenic bodily reaction, one must simultaneously invoke two domains of knowledge. Therefore, an explanation of this phenomenon crosses ontological boundaries and forces the two knowledge domains of body and mind to interact. With regard to young children's reasoning, at least two developmental paths are possible. One possibility is that children will readily accept psychogenic phenomena, either because they fail to honor an ontological distinction between body and mind, or because they honor this distinction but accept that these domains may interact. On this prediction, children should understand psychogenic bodily reactions as readily as any other bodily reactions. This result is plausible, given that even preschool children understand nonobvious causal bases to illness (Kalish, 1996; Siegal, 1988) and contamination (Au, Sidle, \& Rollins, 1993), and probably experience some simple psychosomatic links themselves (e.g., face getting hot when angry). Another possibility is that young children may have particular difficulty understanding psychogenic phenomena, either because these phe-

(C) 2001 by the Society for Research in Child Development, Inc. All rights reserved. 0009-3920/2001/7202-0008 
nomena cross an ontological boundary (i.e., children may think of a disorder as either psychological or bodily, but not both), or because the understanding of complex psychogenic reactions is dependent on other factors (e.g., experience). Surprisingly little is known about children's understanding of psychogenic bodily reactions, yet the developmental story should shed light on the role of ontology in early concepts.

\section{Distinguishing Mental from Bodily Processes}

Wellman and Gelman (1992) argued that children possess common-sense, framework theories (as opposed to scientific, specific theories) that loosely organize their developing understandings. Thus, concepts of physics differ in character, structure, and development from concepts of mental entities and concepts of biology. Most important for this discussion is that causal processes are viewed as distinctly different for physical, mental, and biological entities. Contagion, for example, is a specifically biological process for understanding the transmission of illness. Causal processes that are not biological, such as psychological will or intention, cannot spread disease. Thus, laughter is not contagious in the same way as the flu (Keil, 1992).

Several studies suggest that much of children's knowledge is appropriately domain specific. For example, children appreciate that psychological phenomena are distinct from physical phenomenathoughts and desires are distinct from their physical counterparts (e.g., a thought about a dog versus an actual dog; Wellman, 1990; Wellman \& Estes, 1986). Likewise, preschoolers understand that bodily processes such as breathing or digestion cannot be changed as a result of psychological factors such as desires (Inagaki \& Hatano, 1993; but see Callanan \& Oakes, 1992). Springer and Ruckel (1992) similarly found that preschoolers judged that illness can result from physical but not social factors (e.g., a boy can get sick from eating food that is very old, but not from eating food that was stolen). Schult and Wellman (1997) also found that children appropriately appealed to different causal processes for events from different domains, even when the physical action was identical across domains. For example, even 4-yearolds realized that physical forces and biological factors can counteract intentions or desires and supplied appropriately domain-specific explanations of why the desired event did not occur.

In summary, preschoolers can distinguish between mental states and involuntary biological processes (e.g., preschoolers know that heartbeats are not under conscious control; Inagaki \& Hatano, 1993; Schult \& Wellman, 1997). Past studies, however, have focused on phenomena for which the psychological/bodily distinction is appropriate. In contrast, psychogenic bodily response is of interest for its dual ontology: It is at once both psychological and physical. Thus, how children construe psychogenic bodily response provides an opportunity to determine if and when they invoke the domain distinction, when to do so would be inappropriate.

\section{Illness Understanding}

A selective review of the literature on children's illness understanding was provided to clarify the role of psychogenic bodily reactions in children's illness concepts. Traditionally, it had been thought that children had little understanding of disease and that their understanding was limited by Piagetian stages (e.g., Bibace \& Walsh, 1980). Less cognitively advanced children were thought to engage in magical thinking about illness, whereas more cognitively advanced children were thought to have a more physiological understanding (Perrin \& Gerrity, 1981). Likewise, children were thought to consider illness in terms of immanent justice (e.g., punishment for bad deeds; Kister \& Patterson, 1980).

In contrast to this Piagetian-based literature, various studies have demonstrated that even young children understand several important aspects about illness. Preschoolers understand that illness is a bodily state rather than a psychological state (Springer, 1994) and that illness can have a nonobvious cause (Kalish, 1996; Siegal, 1988). By age 5 children also understand that illness can manifest in nonobvious symptoms (Charman \& Chandiramani, 1995). Although children do at times engage in immanent justice reasoning, researchers suggest it is a default response that children use when they are not yet knowledgeable about the specific causes of certain events (Kister \& Patterson, 1980; Siegal, 1988).

At the same time that children have a generally accurate framework understanding, they lack a detailed understanding of the causes, physiological mechanisms, and manifestations of particular illnesses (e.g., Au \& Romo, 1999). This can lead to confusion about distinctions among various illnesses (Sigelman, Estrada, Derenowski, \& Woods, 1996; Sigelman, Maddock, Epstein, \& Carpenter, 1993). Sigelman et al. (1993), in a survey of fourth through eighth graders and college students, found that the youngest children were least able to differentiate between the causes of three diseases (colds, AIDS, and cancer), and depended on their knowledge of colds and other infectious diseases to inform their understanding of noninfectious diseases, such as cancer. 
The children had many misconceptions on how each disease was transmitted, particularly at the youngest age (see also Charman \& Chandiramani, 1995).

Children's pain concepts are of particular interest in this context, in that pain is both subjective and bodily. Thus, children's understanding of pain provides at least some insight into their reasoning about mind-body links. Some evidence suggests that younger children are less likely than older children to link the sensation of pain with physical bodily states, such as increased pressure in blood vessels (Harbeck \& Peterson, 1992). This finding would seem to suggest that children have some difficulty linking external bodily conditions with internal, subjective states. When given a less demanding task, however, even 5to 6-year-old children accept biological and physical/ behavioral explanations for pain, although at a lower rate than older children (Webb, as cited in Taplin, Goodenough, Webb, \& Vogl, 1999). Children of this age also appreciate that external, physical remedies, such as applying a cream or a band-aid, can be effective means of treating pain (Webb, as cited in Taplin et al., 1999). Thus, by 5 or 6 years of age, children realize that pain is the result of physical actions and events. To the extent that pain is understood as a psychological state, this would seem to be evidence that young children acknowledge that bodily events can affect the mind. The evidence cited does not, however, address the issue of whether pain is construed as men$\mathrm{tal} /$ psychological in the same sense as beliefs, desires, or emotions.

\section{Present Studies}

The present studies examined children's understanding of the interaction of different domains by using psychogenic bodily reactions as a focus of investigation. Psychogenic bodily reactions have several advantages. First, and in contrast to most past work, they are phenomena for which psychological and physical factors truly do interact. Second, psychogenic bodily reactions have a clear empirical basis and are relatively common. Thus, children are likely to have had some experience with them-of their own or of others around them. Third, the components of psychogenic bodily reactions are fairly simple (e.g., feeling worried, getting a stomachache) and likely to be well understood individually even by young children. This contrasts with certain other cross-domain phenomena, such as biofeedback, which would require an understanding of complex components (e.g., the cardiovascular system).

Two caveats are important to keep in mind. First, the studies presented in this article examined interac- tions between mind and body, not interactions between mind and biology. Thus, the data do not speak directly to the current debate regarding whether or not young children have constructed an autonomous domain of biology (e.g., Carey, 1995; Springer, 1996). Nonetheless, the issue of domain interaction is an important problem in its own right. Second, the class of psychogenic phenomena considered in this study is only one of a variety of mind-body links that are possible. Specifically, only cases in which mental states are causes and bodily states are effects were considered, not vice versa (e.g., mind-altering drugs). Furthermore, the focus was on bodily outcomes that are nonconscious, involuntary, and physiological. This contrasts with mind-body links in which the bodily outcomes are conscious, voluntary behaviors; for example, intending to raise your hand (mental cause) leads you to raise your hand (behavioral effect). The present investigation focused on involuntary physiological responses as a starting point because they permitted an especially clear view of domain interactions. That is, involuntary responses themselves have no known psychological component (in contrast to voluntary behaviors, which include an element of psychological intent throughout), and so the extent to which children believed they could be modified by psychological states could be examined.

Several investigators have studied children's illness understanding, but little is known about children's knowledge of psychogenic bodily reactions. The limited evidence that is available suggests that younger children may be less likely than older children or adults to view illness as influenced by psychological and affective events (Bibace \& Walsh, 1980; Burbach \& Peterson, 1986). Only one study (Harris, 1989) focused directly on psychogenic bodily reactions. In this larger study of children's illness concepts, qualitative interviews that included questions about psychogenic bodily reactions were conducted with healthy and chronically ill British children, aged 6 and 10 years. In one section of the open-ended interview, children were asked whether trying to feel a particular emotion could make an illness go away more quickly (or make it get worse). "About one third" of healthy 6-year-olds versus two thirds of healthy 10-year-olds said that psychogenic causality was possible. Meanwhile, "only a handful" of ill 6year-olds and less than one third of ill 10-year-olds said that psychogenic reactions were possible. Harris inferred from these results that experience as well as cognitive maturity were important to children's understanding of the mind-body connection. Why chronically ill children endorsed the possibility of psychogenic effects at a lower rate than healthy chil- 
dren was not clear, but the fact that the ill children were interviewed in a hospital setting while undergoing tests may have adversely affected their performance. The Harris study was a valuable initial investigation into psychogenic understanding. The interview, however, included few questions focusing on psychogenic phenomena (because this was not the focus of the study), and did not include control questions that would enable one to determine how children's responses to psychogenic questions compared with possible responses about other consequences of emotions or other cures for illnesses.

The present studies compared children's understanding of cross-domain, psychogenic events with their understanding of within-domain, psychological or bodily events that included the same components that made up the psychogenic events. Various types of control items were also included to rule out the possibility that children's difficulty with psychogenic items could be attributed to information-processing demands of the task, logical demands of the task, the abstract and hypothetical nature of the questions, or the inclusion of psychological items.

Study 1 investigated the extent to which various age groups of children believed psychogenic bodily reactions were possible. It was predicted that children would not readily acknowledge the possibility of various types of psychogenic bodily reactions, although they would acknowledge other behavioral events involving the same psychological causes, as well as other events involving the same bodily manifestations involving a physical cause.

Because such results could be attributed to lack of experience with psychogenic bodily reactions on the part of the children, Study 2 expanded on Study 1 by testing children's understanding of psychogenic phenomena in a novel situation. Instead of asking children if other children would experience psychogenic bodily reactions (situations in which participants could use their own experience to guide their responses), Study 2 asked children if creatures from another planet could develop a physical response from a psychological cause. Because the bodily reactions in Study 2 were all unknown, participants had minimal experience upon which to base their answers and were able to draw only on their naive understanding of physical and psychological causal processes to help them answer these items. In this more conservative test of children's understanding of psychogenic bodily reactions, the youngest participants were expected to reject psychological causes for novel bodily reactions. Together, this pair of studies provided the first in-depth look at children's beliefs about psychogenic bodily reactions.

\section{STUDY 1}

In this study, children were administered a structured interview in which they were asked whether various physical, psychological, and moral events could cause other physical health and behavioral outcomes. Of primary interest were questions that asked if psychological states could lead a person to have bodily physical reactions (psychogenic bodily reactions). Children in preschool through fifth grade (range = 4,3-11,4) were included to illuminate the developmental patterns. Another interest was whether children's past personal experience with psychogenic bodily reactions would predict their understanding of this phenomenon. Although direct measures of children's experience could not be obtained, self-report questions, as well as a parental questionnaire about children's experiences, were included at the end of the child interview to investigate this question further.

\section{Method}

Participants. Eighty children were recruited from a daycare center and three elementary schools in southeastern Michigan. Four children were dropped from the study because they (1) failed to complete the interview $(n=2),(2)$ answered the practice questions incorrectly $(n=1)$, or (3) answered "yes" to every question $(n=1)$. The remaining participants formed four groups: 18 preschool age $(M=4,9$, range $=4,3-5,3,9$ girls and 9 boys), 27 kindergartners $(M=5,11$, range $=$ $5,5-6,5,13$ girls and 14 boys), 19 second graders ( $M=7,8$, range $=7,3-8,0,7$ girls and 12 boys), and 12 fifth graders $(M=10,8$, range $=10,2-11,4,4$ girls and 8 boys). Children represented a wide variety of socioeconomic backgrounds, and $87 \%$ were identified as White by the interviewer.

In addition, children's parents were given an optional questionnaire to fill out. Parental response rates were $39 \%$ for preschoolers, $70 \%$ for kindergartners, $68 \%$ for second graders, and $50 \%$ for fifth graders. Finally, 28 undergraduate college students from an introductory psychology class at a large midwestern university were included to provide an adult comparison group. An additional 93 college students assisted in pretesting.

Materials and procedure. Structured interviews were administered to children individually by the first author. Before the interviews started, children were informed that they would be told a few stories about other children and then asked some questions, but that there were no right or wrong answers. Children were also given two practice questions to make sure they understood the procedure. The first section of 
the interview included four item types: psychogenic, psychological, physical, and moral. Children were asked to respond yes or no to (1) psychogenic questions: whether a psychological event could cause a physical health outcome (e.g., "Jake felt worried. Do you think Jake would get a tummyache because he felt worried?"), (2) psychological questions: whether a psychological event could cause a behavioral outcome (e.g., "Paul felt worried. Do you think Paul would bite his nails because he felt worried?"), (3) physical questions: whether a physical event could cause a physical health outcome (e.g., "John ate a rotten apple. Do you think John would get a tummyache because he ate a rotten apple?"), and (4) moral questions: whether a moral transgression could cause a physical health outcome (e.g., "Mark didn't clean up after his mess. Do you think Mark would get a tummyache because he didn't clean up after his mess?"). After each question, children were asked to justify their answer ("Why?"). There were five sets of questions; each set included all four item types.

The fact that each set contained all four item types was an important aspect of the study design, because it controlled for participants' willingness to endorse various outcomes and causes. Within each set, the psychological event was kept constant and the physical health outcome was kept constant. In the example set provided earlier, the physical health outcome was always getting a stomachache, and the psychological event was feeling worried (both times). This design meant that if in fact children had difficulty with the psychogenic items, that difficulty was not due to either component alone (feeling worried or getting a stomachache), but rather to the two in conjunction. Thus, if children performed well on the psychological and physical items, as predicted, a lack of understanding in the psychogenic case could not be explained by a misunderstanding of the causes or consequences asked about in the scenario.

Moral items were included so that there would be one question in each set for which the correct answer was clearly "no" (see Siegal, 1988, for evidence that children recognize that moral transgressions cannot cause disease). This was useful to differentiate a pattern of correct understanding from simply a bias toward answering "yes." Finally, other biases were determined on the basis of the overall pattern of a child's answers. Some children might not endorse physical, moral, and psychogenic items, for instance, because they refuse to allow "negative" outcomes.

Questions were administered in a different random order for each child, with no two questions from the same set or of the same type in a row (except the last item in a few instances). The gender of the story characters in each question remained consistent throughout the interview and corresponded to the gender of the participant.

The final portion of the child interview asked about the child's own experience with the bodily reactions presented in the first part of the interview (e.g., "Have you ever gotten a tummyache before?" or "Have you ever gotten a tummyache because you felt worried?"). In this series of yes-no questions, if the child answered "no" to ever having the bodily reactions, the psychogenic experience question was omitted and automatically coded as "no." These questions were asked in the same order for all participants. To help validate children's answers for the experiential questions, an optional survey was sent home to all parents whose children were interviewed.

Pretesting. The items were selected on the basis of college students' answers to similar items on a selfadministered questionnaire. Adults were tested in a group setting. Introductory psychology students $(N=$ 93) were asked to rate 52 statements (all four item types of 13 sets; e.g., "Eating a rotten apple can lead a person to have a tummyache") on a 6-point Likert scale, from 1 (strongly disagree) to 6 (strongly agree). On the basis of these ratings, five sets were retained for which moral statements were judged low (mean of each $<3$ ), and physical, psychological, and psychogenic statements were judged high (mean of each $>4$ ).

After some minor revisions, the remaining 20 statements were rated by an additional 28 adults (see Table 1$)$. Their ratings of psychogenic $(M=4.38)$, psychological $(M=4.68)$, and physical items $(M=4.64)$ did not differ significantly from one another (with a significance level of .008 to control for chance on six comparisons), whereas the mean for moral $(M=2.45)$ items was significantly lower than each of the other three types, $t \mathrm{~s}(27)>15, p s<.001$. Thus, adults typically endorsed the psychological, physical, and psychogenic links and rejected the moral links.

\section{Results}

For each age group, children's responses were averaged to get the percentage of "correct" versus "incorrect" answers for each type. "Yes" responses to psychogenic, psychological, and physical items were considered correct, and "no" responses to moral items were considered correct. Adult Likert scale ratings were converted to "yes" and "no" answers so that adults could be included in the developmental analyses. A rating of 1,2 , or 3 was assigned a "No" and a rating of 4,5 , or 6 was assigned a "Yes." A frequency analysis (with moral items reverse coded) showed that the modal adult response was a 5 across 
Table 1 Adult Mean Acceptance Ratings for Each Item in Study 1 ( range = 1-6)

\begin{tabular}{llll}
\hline Item Type & & \multicolumn{1}{c}{ Cause } & Mean Acceptance Rating \\
\hline Psychogenic & Feel nervous & Throw up & $4.57(.79)$ \\
Psychological & Feel nervous & Jiggle legs & $4.79(.63)$ \\
Physical & Eat too much candy & Throw up & $4.39(.69)$ \\
Moral & Pull a cat's tail & Throw up & $1.25(.44)$ \\
Psychogenic & Feel worried & Have a tummyache & $4.64(.91)$ \\
Psychological & Feel worried & Bite nails & $4.71(.90)$ \\
Physical & Eat a rotten apple & Have a tummyache & $5.18(.67)$ \\
Moral & Make a mess and not clean it up & Have a tummyache & $2.00(1.09)$ \\
Psychogenic & Feel scared & Have goosebumps & $3.82(1.31)$ \\
Psychological & Feel scared & Scream & $4.82(.72)$ \\
Physical & Eat ice cream on cold day & Have goosebumps & $4.71(1.05)$ \\
Moral & Tell a lie & Have goosebumps & $2.54(1.14)$ \\
Psychogenic & Feel frustrated & Have a headache & $4.79(1.10)$ \\
Psychological & Feel frustrated & Throw something & $4.36(.87)$ \\
Physical & Sneezed on by someone with flu germs & Have a headache & $3.32(1.22)$ \\
Moral & Punch someone & Have a headache & $3.07(1.18)$ \\
Psychogenic & Feel shy & Face gets red & $4.07(.96)$ \\
Psychological & Feel shy & Become quiet & $4.71(1.58)$ \\
Physical & Get the chicken pox & Kick a dog & Face gets red in places \\
Moral & Face gets red & $5.61(.50)$ \\
\hline
\end{tabular}

Note: Standard deviations are in parentheses.

all items. In fact, $59 \%$ of all responses were a 5 or a 6 . For 15 out of 20 of the items, the modal response was a 5 or 6 . These results seem to support the decision to collapse the adult rating scale down to 2 points (correct or incorrect).

Preliminary analyses revealed no main effects for gender, nor any interpretable interactions involving gender. Therefore, this variable was excluded from all further analyses. A 5 (age) $\times 4$ (item type) repeatedmeasures ANOVA was conducted, with age (preschool, kindergarten, second grade, fifth grade, adult) as the between-subjects variable and item type (physical, psychological, moral, and psychogenic) as the within-subjects variable. Table 2 presents the percentage of correct responses for each age group by item type.

A main effect was found for age, $F(4,99)=7.13$, $p<.001$. Adults had a significantly higher percentage of correct answers than all other age groups. Pre- schoolers, kindergartners, second graders, and fifth graders did not differ from one another in overall performance. A main effect was also found for item type, $F(3,297)=31.65, p<.001$. Psychogenic items yielded lower scores than all other item types, $p<.01$. Scores on the psychological, physical, and moral items did not differ significantly from one another.

In addition to the main effects just described, a significant Age $\times$ Item Type interaction was found, $F(12,297)=4.60, p<.001$, which indicated a large improvement with age on the psychogenic items, $p<$ .001, simple-effects test. Adults performed better than all other age groups, $p<.01$, Newman-Keuls. Improvement with age on the psychological items was also found, $p<.005$, simple-effects test, with adults performing better than preschoolers, kindergartners, and fifth graders, $p<.05$, Newman-Keuls, but not second graders.

Table 2 Study 1, Mean Percentage of Correct Responses, by Item Type and Age

\begin{tabular}{lccccc}
\hline Item Type & Preschool & Kindergarten & Grade 2 & Grade 5 & Adult \\
\hline Psychogenic & $38(34)_{\mathrm{a}}$ & $42(39)_{\mathrm{a}}$ & $44(29)_{\mathrm{a}}$ & $60(23)_{\mathrm{a}}$ & $86(16)_{\mathrm{a}}$ \\
Psychological & $67(28)_{\mathrm{b}}$ & $77(23)_{\mathrm{b}}$ & $83(14)_{\mathrm{b}}$ & $72(22)$ & $94(13)_{\mathrm{a}}$ \\
Physical & $81(21)_{\mathrm{b}}$ & $82(20)_{\mathrm{b}}$ & $82(16)_{\mathrm{b}}$ & $77(22)$ & $88(13)_{\mathrm{a}}$ \\
Moral & $78(29)_{\mathrm{b}}$ & $79(23)_{\mathrm{b}}$ & $88(14)_{\mathrm{b}}$ & $88(13)_{\mathrm{b}}$ & $71(21)_{\mathrm{b}}$ \\
\hline
\end{tabular}

Note: Within each age group, columns with different subscripts are significantly different from one another at the .05 level. Standard deviations are in parentheses. 
Table 3 Study 1, Percentage of Participants Classified in Each Response Pattern, as a Function of Age

\begin{tabular}{lcccrr}
\hline Response Pattern & Preschool & Kindergarten & Grade 2 & Grade 5 & Adult \\
\hline Adult (correct) & $22(4)$ & $26(7)$ & $32(6)$ & $67(8)$ & $79(22)$ \\
Low psychogenic & $33(6)$ & $37(10)$ & $63(12)$ & $8(1)$ & $0(0)$ \\
Low emotional & $11(2)$ & $15(4)$ & $0(0)$ & $8(1)$ & $0(0)$ \\
Avoidance of negative outcomes & $0(0)$ & $7(2)$ & $5(1)$ & $8(1)$ & $0(0)$ \\
Bias toward responding "yes" & $11(2)$ & $15(4)$ & $0(0)$ & $0(0)$ & $18(5)$ \\
Bias toward responding "no" & $11(2)$ & $0(0)$ & $0(0)$ & $8(1)$ & $0(0)$ \\
Other & $11(2)$ & $0(0)$ & $0(0)$ & $0(0)$ & $4(1)$ \\
\hline
\end{tabular}

Note: $n$ s are in parentheses.

Patterns of responding. To investigate individual patterns in responding, each participant was classified into one of six meaningful response patterns or "other" (see Table 3). Participants' moral, psychogenic, psychological, and physical scores were each rated as either high ( $\geq 50 \%$ correct) or low $(\leq 49 \%$ correct). These four ratings formed 16 possible combinations, 6 of which were identified a priori as meaningful. The meaningful patterns included (1) the adult (correct) pattern, in which respondents were high on all four types; (2) the low psychogenic pattern, in which respondents were low on psychogenic, but high on the other three types; (3) the low emotional pattern, in which respondents were low on psychogenic and psychological, and high on physical and moral; (4) the avoidance of negative outcomes pattern, in which respondents were high on moral and psychological, but low on psychogenic and physical; (5) the bias toward responding "yes" pattern, in which participants were low on moral, but high on the other three types; and (6) the bias toward responding "no" pattern, in which respondents were high on moral, but low on the other three types. Results showed that the modal majority of the preschoolers, kindergartners, and second graders were classified in the low psychogenic pattern, whereas the majority of the fifth graders and adults were classified in the adult (correct) pattern.

Item analyses. To determine the generality of these effects, each of the five item sets (i.e., the five sets of questions each including all four item types) was examined individually. The results mirrored those for the aggregate data. Most strikingly, psychogenic items consistently yielded poor performance among the children. Each of the five item sets was also examined for each of the four age groups of children, to see which of the four item types (psychogenic, psychological, physical, or moral) elicited the lowest level of performance within the set. By chance alone, psychogenic items was to score lowest on one fourth of the sets, for a total of five sets. Psychogenic items, however, elicited the lowest level of performance for 14 of the 20 item sets ( 5 for preschoolers, 4 for kindergartners, 3 for second graders, and 2 for fifth graders). For five of the six remaining item sets, the psychogenic item was either tied for the lowest score or within 5 percentage points of the lowest score. In contrast, for adults, psychogenic items scored lowest in only one item set (out of five).

Explanations. Children's explanations could provide additional insight into their reasoning, either in support of the patterns from the yes / no responses, or in contrast to those results. If older children were better able than younger children to support their correct answers with psychogenic explanations, this would lend further support to the developmental comparisons. In contrast, if a child answered "no" to the psychogenic question, but gave a reason that indicated that the psychogenic effect was possible or that another bodily reaction could result from a psychological cause, then this would indicate that psychogenic reasoning was not adequately assessed with the yes / no response format. Finally, children's explanations could shed light on the nature of their reasoning.

For each psychogenic question, children's explanations were scored as either showing or not showing understanding of psychogenic bodily reactions. (Adults could not be included in this analysis because they were not asked to explain their answers.) On the question regarding blushing after feeling shy, for example, an explanation would be scored as showing understanding of psychogenic bodily reactions if a child said, "Sometimes you blush when you are shy or embarrassed." A nonpsychogenic explanation to the same item included, "Because she's shy," "That wouldn't happen," or "I don't know." Note that children's explanations were scored independently of their yes/no responses. Thus, it was possible for an incorrect yes / no answer to be followed by an explanation that demonstrated psychogenic reasoning. Conversely, a correct yes/no answer could be followed by an explanation that did not demonstrate 
Table 4 Study 1, Percentage of Trials with Explanations Showing Evidence for Psychogenic Reasoning and Not Showing Evidence for Psychogenic Reasoning, as a Function of Age and Yes/No Answer

\begin{tabular}{lcccc}
\hline & Preschool & Kindergarten & Grade 2 & Grade 5 \\
\hline Yes/no answer correct & & & & \\
$\quad$ Psychogenic explanation & $18(6)$ & $28(16)$ & $55(23)$ & $81(29)$ \\
$\quad$ Nonpsychogenic explanations & $82(28)$ & $72(41)$ & $45(19)$ & $19(7)$ \\
Yes/no answer incorrect & & & & \\
$\quad$ Psychogenic explanation & $5(3)$ & $6(5)$ & $6(3)$ & $21(5)$ \\
$\quad$ Nonpsychogenic explanation & $95(53)$ & $94(73)$ & $94(50)$ & $79(19)$ \\
Appeal to experience & $3(3)$ & $3(4)$ & $8(8)$ & $10(6)$ \\
\hline
\end{tabular}

Note: $n$ s are in parentheses.

psychogenic reasoning. Answers that referred to either personal experience or the experience of a family member (e.g., "my mother gets this (a headache) when I annoy her") were also coded. Two coders rated children's responses. One coder rated all of the transcripts; the second coder rated $26 \%(N=20)$ of the transcripts, randomly selected. Agreement between the two coders was $94 \%$.

As can be seen in Table 4, psychogenic explanations were most frequent on those trials for which participants provided a correct yes/no answer. Even so, psychogenic explanations increased over age; correspondingly, nonpsychogenic explanations decreased. Although the percentage of time that respondents appealed to experience increased slightly with age, this was quite modest, and nowhere near the rate of increase of psychogenic explanations overall. This suggests that developmental changes that are beyond the effects of increased ability to retrieve memory of a past psychogenic event may be occurring in children's understanding. A final point is that the forcedchoice task seemed to be fairly accurate in crediting children with psychogenic understanding, given the low percentage of children scored as incorrect on the yes/no task who demonstrated good psychogenic reasoning in their explanations.

Although this study was not designed to examine the possibility that children treated psychogenic events as biological, children's justifications provided an opportunity to examine this issue indirectly. That is, children's justifications on those trials for which they denied a psychological event were examined to determine how often they invoked an alternative cause that was biological in nature. To the extent that children did invoke biological alternative causes, this would suggest that they were construing these events as biological (not merely bodily). To conduct this analysis, all alternative-cause justifications given on trials where children denied a psychogenic effect were first identified. Each justification was then coded as belonging to one of five categories: biological (illness, digestion, elimination; e.g., "Only happens when you're sick"), environmental (e.g., "Only if it's cold"), sensation (e.g., "She wasn't hot"), psychological ("Only happens if you're excited"), or other (e.g., "She wasn't crying").

There were 158 justifications for "no" answers to psychogenic questions. Three coders identified 28\% of these ( $n=45$, across the four child age groups) as alternative causes (average agreement among the three coders was 94\%). Average agreement on classification of these 45 justifications into the five coding categories was $83 \%$, and all disagreements were resolved by discussion. Results of this analysis provided preliminary support for the claim that children hold a biological-cause model. Specifically, the modal alternative-cause justification focused on potential biological causes (58\% biological, 16\% environmental, $18 \%$ sensation, $2 \%$ psychological, and $7 \%$ other). This pattern was found in each of the four age groups.

Reports of personal experience. Children's self-reported psychogenic experiences were summed and entered into a one-way ANOVA, with age as the dependent variable. A main effect was found for age, $F(3,72)=$ $4.13, p<.01$, which indicated that fifth graders reported higher incidences of psychogenic bodily reactions $(M=2.17$ out of 5$)$ than each of the younger age groups (Ms ranging from 0.83 to 1.29 ), $p s<.05$, Newman-Keuls. A similar developmental trend was found when those trials on which children reported not having experienced one or more of these bodily conditions (tummyache, headache, throwing up, goosebumps, or face turning red) were dropped. That is, each child received a percentage score ranging from 0 to 1 , which reflected the number of trials (out of five) on which they reported a psychogenic bodily reaction, divided by the number of trials on which they reported ever having experienced the bodily condition (with or without a psychological cause). In this analysis, a main effect was again found for age, 
$F(3,70)=3.01, p<.05$, with fifth graders scoring higher than second graders, kindergartners, and preschoolers ( $M s=.48, .28, .24$, and .22 , respectively), $p \mathrm{~s}<.05$, Newman-Keuls.

Children's self-reported personal experiences with psychogenic bodily reactions were correlated with their performance on the corresponding psychogenic items, after partialling out the effects of age. A positive association was found between children's self-reported experience with, and their understanding of, psychogenically caused bodily reactions for each of the five items considered individually, $r s$ of .34 to .41 , all $p$ s $<.01$. In addition, children were divided into two groups, one of which reported experience with psychogenic bodily reactions on at least one of the five trials $(n=44)$ and the other of which did not $(n=32)$. The mean scores on psychogenic items for the two groups were compared by using a simple factorial ANCOVA, with age as the covariate. The results show that the group that had reported experience with psychogenic bodily reactions scored significantly higher on psychogenic items (.58) than the group that had reported no experience with psychogenic bodily reactions, .26, $F(1,75)=17.13, p<.001$.

When the same analyses were conducted by using parental reports of their child's experience with psychogenic bodily reactions, no relationships were found. In fact, parent and child reports of experience were not significantly related. How often parents said they discussed psychogenic bodily reactions with their children was also examined, and it was found that this rate did not change significantly with age: $14 \%$ in preschool, 37\% in kindergarten, $69 \%$ in second grade, and $50 \%$ in fifth grade. A simple factorial ANCOVA, using age as the covariate, did not distinguish psychogenic performance for those children who were reported to have discussed psychogenic bodily reactions with their parents versus the remaining children.

\section{Discussion}

The primary finding of Study 1 was that children from preschool age through fifth grade were much less likely to accept psychogenic bodily reactions than adults. This result was found when data were collapsed across items, when items were examined individually, and when participants were classified into response patterns. The developmental pattern was found both with participants' yes/no responses and with their justifications. A second, related finding was that children were less likely to accept psychogenic bodily reactions than either physically caused bodily reactions (physical items) or psychologically caused behavioral reactions (psychological items). This was so even though the psychological causes were identical for the psychological and the psychogenic items, and the physical outcomes were identical for the physical and the psychogenic items.

It was hypothesized that children might be particularly resistant to the psychogenic items because they tap into cross-domain understanding. That is, children's difficulty might lie precisely in accepting that a psychological cause could have a bodily effect. Such an explanation would account for children's high performance on the within-domain items (physical and psychological). Alternative explanations of these data, however, are possible.

One possibility is that age differences on this task were due to increased information-processing abilities. Older children might have a better grasp of the elements of the task (e.g., phrases such as "felt frustrated" or "flu germs") or might be better at mentally retaining both cause and effect on a given item. Perhaps the younger children were biased toward responding "no" to every item. The design of the study and the obtained patterns of results, however, argue against these explanations. Even the youngest children had no difficulty reasoning about the components of the task (e.g., feeling frustrated as a cause; getting a headache as an effect) when they were presented on the physical and psychological items. Moreover, the pattern displayed by the younger children (poor performance on psychogenic, high on the remainder) could be found only if they were able to mentally retain both cause and effect. If children had focused on causes only, they would have rejected both psychogenic and psychological items; if they had focused on effects only, they would have rejected psychogenic, physical, and moral items. Instead, children selectively rejected psychogenic items. Children also had no difficulty appropriately supplying both "yes" and "no" responses, as indicated by their responses to physical, psychological, and moral questions (which required "yes," "yes," and "no" answers, respectively, to be coded as correct).

A second alternative account of the results is that children's responses might reflect their belief that psychogenic reactions are relatively rare. When children were asked to judge whether an event occurred, perhaps they were judging the probability of its occurrence rather than the possibility of its occurrence. A child might reason that worrying could lead to a stomachache, but for any given individual this effect would be rare, so the child predicted that it did not happen for the character in the story. According to this view, children did not deny that psychogenic 
bodily effects could take place, but rather judged them to be infrequent. This interpretation cannot, however, fully explain the results, because a number of the other items were likewise relatively rare (e.g., biting nails when worried, throwing something when frustrated, jiggling legs when nervous), but were readily accepted by even the youngest children. It is also difficult to see how this would account for the developmental differences that were found. Nonetheless, Study 2 was designed in part to provide further evidence regarding this alternative interpretation. In Study 2, all events were novel and thus low probability.

A third possibility is that children's difficulty with psychogenic items arose from their own lack of personal experience with these events, rather than a resistance to cross-domain phenomena. In support of this interpretation, children's self-reported personal experiences with psychogenic bodily reactions did correlate significantly with their rate of accepting psychogenic phenomena. Respondents who reported any type of experience with psychogenic bodily reactions scored higher on the psychogenic items than those with no experience. Unfortunately, however, causal direction could not be determined: Understanding of psychogenic phenomena could have developed as a result of personal experience, or understanding of one's own psychogenic experiences could have developed as a result of coming to appreciate psychogenic phenomena more generally. To illustrate the latter: Young children who get stomachaches when worried may not link the emotional state with the bodily state, and may simply believe that they are sick. It is also possible that the correlation between the two measures was inflated because the selfreport questions directly followed the primary study questions.

Complicating this issue further is the finding that parental reports about children's psychogenic experiences did not correlate significantly with children's reports of their own psychogenic experiences, nor were parental reports associated with children's performance on psychogenic items. This lack of correspondence does not indicate who is more accurate: children or parents. On the one hand, it could simply show that the parents had little direct access to their children's emotional states (i.e., that the children were more accurate). On the other hand, it could indicate that the young children were not very sensitive to their own psychogenic experiences (i.e., that the parents were more accurate). It is important, however, to keep in mind that the number of parents who filled out and returned the questionnaire was quite small (7 parents of preschoolers, 18 of kindergartners, 8 of second graders, and 6 of fifth graders), so confidence in these results is limited. One indirect piece of evidence that children's responses do not simply reflect lack of experience is that the youngest children were likely to have less experience with all of these items (e.g., getting a headache because of the flu), but they were lower only on the psychogenic items.

In summary, Study 1 demonstrates that an understanding of psychogenic bodily reactions increased rather dramatically with age. The design of the study argues against the possibility that age differences were due to general task demands, because the age effects were specific to psychogenic reasoning. It is suggested that these results might reflect children's tendency to distinguish the domains of psychology and bodily experience. Study 1 left unclear, however, the role of experience in mediating psychogenic understanding. It is also possible that children were reporting their beliefs about event frequency rather than event probability. Study 2 addressed these issues more directly.

\section{STUDY 2}

Study 2 tested children's understanding of psychogenic bodily reactions when prior experience was minimized. To reduce the effects of participants' prior experience, study questions that concerned hypothetical creatures from another planet were posed. For instance, on one item children heard, "Plunim has two antennae. One day, Plunim's antennae drooped. Do you think Plunim's antennae drooped because she felt angry [psychological cause] or because she got pricked with a dirty needle [physical cause]?" Because children did not have any familiarity with what happens on Plunim's planet, they were forced to invoke their naive biases regarding psychological and physical (bodily) causal processes. Even more important, with no reference to situations they might have experienced before, participants' responses revealed whether they believed that mental states could influence bodily reactions. The rationale for using a forcedchoice response format in Study 2 (versus a yes/no response format in Study 1) was to avoid a "floor effect," with respondents rejecting all scenarios simply because they did not occur in real life. The forcedchoice format also allowed assessment of the relative strength of psychological versus physical causes of bodily events.

Study 2 had several advantages that built on Study 1. First, Study 2 helped to determine whether the developmental differences shown in Study 1 were the direct result of knowledge/experience with particular psychogenic events. If past experience was the prime determinant, then no age differences in perfor- 
mance would be observed in Study 2, for which children could not rely on past experience. Second, Study 2 allowed for the examination of the generality of children's understanding, when they began to endorse psychogenic bodily reactions. If children had a general appreciation for the phenomenon, then they would invoke it even with novel examples.

Third, Study 2 helped to circumvent some of the problems with the yes / no format of Study 1, in which young participants may have rejected certain items because they were rare. Because all of the events in Study 2 were unlikely (i.e., anything resulting in drooping antennae was outside the child's experience), children would not select their answers on the basis of which events were familiar. Furthermore, the psychological causes (various common emotional states) were considerably more frequent in children's past experience than the physical causes (various unpleasant mishaps) so that if the younger children were relying on probability of occurrence, in Study 2 they would have a preference for endorsing the psychogenic scenarios (psychological causes of the bodily reactions). If, however, they had a principled avoidance of psychogenic events, they would have a preference for endorsing the physical scenarios (physical causes of the bodily reactions).

\section{Methods}

Participants. Fifty-four children were recruited from three daycare centers and two public elementary schools in southeastern Michigan. None of these children had participated in Study 1. Participants formed three groups: 18 preschoolers (mean age $=$ 4,6 , range $=4,3-5,0,9$ girls and 9 boys), 18 kindergartners (mean age $=5,7$, range $=5,5-6,4,8$ girls and 10 boys), and 18 second graders (mean age $=7,8$, range $=$ $7,3-8,0,8$ girls and 10 boys). Children represented a wide variety of socioeconomic backgrounds, and $96 \%$ were identified as White by the interviewer.
In addition, 48 undergraduates from an introductory psychology class at a large midwestern university were recruited to pretest the items. Their data served as the adult comparison group in the analyses.

Materials and procedure. All interviews occurred in a private research room and lasted approximately 15 min. Children heard the following introductory statement: "Zantar is an alien planet. All the creatures on planet Zantar are just like people, but their bodies look very different. Listen to the stories and look at the pictures about the beings on planet Zantar and after each story, tell me which answer you think is best." After two easy practice questions (e.g., would someone who received birthday presents open them up or throw them away?), intended to check that participants were comfortable and attentive to the task, eight questions were presented to each child.

Each question first presented a character (matched in gender to the participant) and a particular attribute of that character (e.g., "Tarlin has a tail"). Next, participants were told that some change had occurred in that attribute (e.g., "One day, Tarlin's tail hurt"). Finally, participants were asked to choose between two possibilities (psychological or physical) to explain why this change in the attribute had occurred (e.g., "Do you think Tarlin's tail hurt because $\mathrm{s} /$ he felt worried [psychological choice] or because $\mathrm{s} /$ he ate something that was rotten [physical choice]?").

A list of items appears in Table 5. A book of simple illustrations accompanied the questions. The different creatures looked alike except that the relevant attribute for each character was highlighted (crosshatched, bolded, colored) in that character's illustration. In addition, a before-and-after picture was presented to further highlight the particular change in each attribute.

After answering the question, participants were given the opportunity to explain their choice (“Why?").

Table 5 Study 2 Items

\begin{tabular}{lll}
\hline Bodily Change & Psychological Cause & \multicolumn{1}{c}{ Physical Cause } \\
\hline Tail hurt & Felt worried & Ate something that was rotten \\
Claws hurt & Felt frustrated & Was touched by someone with germs \\
Antennae drooped & Felt angry & Got pricked with a dirty needle \\
Eyebrows got white stripes & Felt mad & Was bitten by a bug \\
Toes got puffy & Felt embarrassed & Drank dirty water \\
Red eyes turned yellow & Felt frightened & Was scratched by an animal \\
Tongue ached & Felt disappointed & Sat in the sun too long \\
Teeth stung & Felt jealous & Got someone else's blood mixed in \\
& & his/her blood \\
\hline
\end{tabular}


Items were randomly ordered for each participant. The order of answer choice (psychological or physical) within each item alternated over the set of questions for each participant.

Pretesting. Items were designed to be analogous to real-life psychogenic and physical bodily reactions but to differ in the specifics to discourage drawing on human experience to answer the questions. Thus, for example, participants were not asked whether eating something cold would make an alien's fur stand up on its back, because this would be too similar to actual human experience. The goal was to come up with a set of items for which both psychological and physical answers would be appropriate (as judged by adults), so as to enable observation of children's naive biases regarding illness causality. Thus, items were selected so that half the time adult participants would choose psychological reasons and half the time they would choose physical reasons.

Forty-eight undergraduates were administered a questionnaire version of the Study 2 interview, with a larger set of items. Physical answers were coded as 0 and psychological answers were coded as 1, and the average response was calculated for each participant. The mean across participants was .44, which indicated that participants were equally likely to choose physical and psychological responses. Of this larger set of items, eight items were selected that individually showed no bias toward either physical or psychological responses (i.e., the average response across participants for each question fell between .35 and .64). The average total score on these eight items on the pretesting questionnaire was .49 .

\section{Results}

As with the pretest, each physical answer was coded as 0 and each psychological answer was coded as 1 . Although preliminary analyses included gender as a between-subjects variable, this variable was excluded from final analyses because there were no interpretable effects involving gender. Answers were averaged across the eight items and entered into a one-way repeated-measures ANOVA, with age (preschool, kindergarten, second grade, adult) as the between-subjects variable.

A main effect was found for age, $F(3,98)=9.35$, $p<.001$, which indicates that preschoolers $(M=.21)$ and kindergartners $(M=.24)$ chose fewer psychological responses than second graders $(M=.54)$ or adults $(M=.49), p s<.01$, by Newman-Keuls post-hoc tests. No differences in responses were found between preschoolers and kindergartners or between second graders and adults. Preschoolers and kindergartners selected psychological responses below chance, $p$ s $<$ .001 by $t$ test, whereas second graders' and adults' choices did not differ from chance.

An examination of individual items mirrors the results of the ANOVA, namely, a pattern of avoiding psychological responses among preschoolers and kindergartners and a mixed response pattern among second graders. Specifically, both preschoolers and kindergartners selected the psychological choice less than $30 \%$ of the time on seven of the eight items, but second graders did not show this pattern for any of the eight items. Whereas seven of the eight items scored close to the midpoint for second graders (i.e., between $35 \%-65 \%$ ), only one item at each of the preschool and kindergarten ages scored in that middle range.

In addition, the response patterns of individuals were examined. Participants at each age were individually classified into three groups: One group that chose primarily physical causes (six to eight trials out of eight), another group that chose primarily psychological causes (six to eight out of eight), and a third group that chose a mixture of both physical and psychological causes (all remaining participants). The results of this analysis were consistent with the ANOVA analyses: Primarily physical causes decreased with age $(72 \%$ of preschoolers and $67 \%$ of kindergartners versus $28 \%$ of second graders and $25 \%$ of adults), and primarily psychological causes increased with age (0\% of preschoolers and $5 \%$ of kindergartners versus $39 \%$ of second graders and $23 \%$ of adults).

\section{Discussion}

Study 2 showed a clear developmental shift between kindergarten and second grade in children's willingness to attribute psychological causes to novel bodily events. When asked what could have caused various bodily reactions in alien creatures, adults and second graders selected both psychological and physical causes, whereas preschoolers and kindergartners strongly preferred physical causes. These results were consistent with those of Study 1. As in Study 1, the youngest children seemed to avoid psychogenic responses, and this tendency diminished with age.

The present results also extend beyond those of the first study. Because the questions in Study 2 concerned unfamiliar alien creatures, participants could not rely on their knowledge of particular past events that they or others had experienced. In other words, the pattern of results cannot be attributed to a simple response strategy in which participants endorsed those events that they had heard of before, and re- 
jected those events that they had not heard of before. Instead, participants were differentiating the events at a more abstract level. Furthermore, because the psychological causes (i.e., simple emotions) were more commonly experienced than the physical causes (i.e., unusual mishaps), avoiding a psychogenic response entailed selecting the less common event. Because the youngest children preferred the physical causes, they were not simply selecting causes on the basis of their familiarity or probability of occurrence. This further supports the interpretation that younger children do not simply report those events with which they are familiar, but rather seem to reject cross-domain scenarios.

At the same time, the data cannot be used to argue that past experience played no role in children's understanding of these events. It may be that the children had come to deny the possibility of psychogenic events because they rarely experienced them, even though these specific bodily events were unfamiliar to all participants. That is, the children may in effect have been saying "I've never seen that kind of event [psychogenic], but I have seen that kind of event [physical]." This could be thought of as a more subtle form of experience-based responding. If, however, this was in fact what children were doing, it demonstrates that children had gone from experiencing a selected set of specific events to forming abstract generalizations about which sorts of events were possible or not. This possibility is considered in the General Discussion.

One difference in the results of the two studies is that second graders endorsed psychogenic causes in Study 2, but not in Study 1. This discrepancy could reflect the different task demands across the two studies. In both studies, second graders accepted psychogenic events on roughly half the trials. In Study 1, this rate of response was considered low because it fell far below response rates on corresponding psychological or physical items. In Study 2 , however, this rate of response was considered relatively high because it far exceeded the rates of preschoolers and kindergartners. In any case, it is clear that the youngest children (preschoolers and kindergartners) consistently denied psychogenic bodily reactions, that second and fifth graders were in a period of transition, and that adults readily accepted psychogenic events.

\section{GENERAL DISCUSSION}

The results of these two studies support a developmental path from viewing psychogenic bodily responses as wholly physical phenomena to viewing them as both physical and psychological phenomena. Young children typically denied that psychogenic bodily reactions could occur and predicted that unfamiliar bodily conditions resulted from physical causes only. Adults, however, typically reported that psychogenic bodily reactions were possible and that unfamiliar bodily conditions could result from either psychological or physical causes. Children's responses in both studies were consistent with the interpretation that they believed that bodily ailments could not result from a mental cause. These results extend beyond recent findings showing that children distinguished mental from physical phenomena when appropriate (see Hatano \& Inagaki, 1994; Schult \& Wellman, 1997; Wellman \& Estes, 1986). Even when considering phenomena in which the domains did in fact interact, young children continued to deny crossdomain interactions.

One issue that this research leaves open concerns the nature of children's biological understandings. Although the present data suggest that children distinguished mind from body, whether they construed these bodily conditions as biological is unclear. Carey (1995) and Au and Romo (1999) argued that young children's grasp of bodily events are not at first biological, whereas Keil $(1992,1994)$ and Springer (1992, 1996) argued that they are biological. This debate will not be recapitulated here, but Kalish's (1997) point that what "counts" as biological understanding will depend in part on one's criterion (e.g., possessing domain-specific facts versus specific understanding of the mechanisms involved in the causal processes is emphasized).

Another question concerns the generality of the current results. Psychogenic bodily reactions are just one example of cross-domain phenomena, and it is not known if other cross-domain phenomena similarly pose conceptual difficulties for children. Future research could test children's understanding of other mind-body concepts such as biofeedback, meditation/hypnosis, or enhanced bodily performance. One could also extend this research design to examine phenomena in which a physical cause (e.g., a drug) results in a psychological effect (e.g., changed mental state). In all of these cases, the theoretical account presented here would predict that young children would have difficulty understanding these events as crossdomain phenomena. Until more data are available, however, the present studies must be understood as a demonstration that children do not recognize one case of mind-body interaction (i.e., psychogenic bodily reactions), rather than as evidence supporting the hypothesis that young children are reluctant to cross domain boundaries. 
Intentional behaviors may also be argued to involve domain crossing but certainly are readily accepted by even young children. For example, intentionally carrying out an action, such as deciding to walk across a room, could be argued to entail a psychological cause (one's intention) and a physical effect (the physical behavior of moving one's body through space). If accepting these cases entails transcending domain boundaries, then this would undercut the theoretical explanation thus far provided. The apparent conflict, however, between the theoretical account presented here and this class of phenomenon is a true conflict only if children in fact view volitional behaviors as instances of mind-body interaction. This is an empirical question that has not yet been tested. Children might accept the contingency between mental states and intentional actions (on the basis of extensive personal experience) without considering the causal implications of such phenomena (e.g., analogous to how children understand remotecontrol devices).

Alternatively, it is possible that children do understand that with volitional behaviors, mental states can cause bodily outcomes. If so, then a more specific explanation would be required to explain the difficulty that children had with the phenomena examined in the present studies. Children's difficulty, for example, may specifically involve understanding that mental states can cause involuntary bodily responses. Thus, children's difficulty may have been with mindbiology interactions, rather than with mind-body interactions.

One crucial issue in this work concerns the source of the developmental differences that were obtained, including the role of experience. Although Study 2 rules out the possibility that developmental differences were simply due to differing levels of familiarity with specific psychogenic phenomena, experience is certainly one plausible source of developmental change in this area. The key issue is whether children denied cross-domain interactions (1) in spite of encountering evidence to the contrary, or instead (2) because they had failed to encounter sufficient evidence for them. From a practical or applied perspective, the answer to this question is important in determining how to help younger children appreciate their own psychogenic bodily reactions and potentially aid in their own care. From a theoretical perspective, the answer is important in understanding the role of domains in early cognition.

The present data do not provide direct evidence on this issue. Nor would it be straightforward to examine children's own experiences of psychogenic reactions, because parents do not have direct access to children's emotional states, and children's selfreports may be limited by their own conceptual biases (e.g., a child may experience a stress-induced headache, yet not realize the true cause because he or she believes that mental states cannot affect the body). It would be valuable, however, to conduct an intervention study to determine how readily children can be taught to appreciate cross-domain (specifically, psychogenic) phenomena. If indeed children have a conceptual bias to resist cross-domain interactions, then direct training should have little effect on children of kindergarten age or younger. If children are limited instead by lack of knowledge and experience, then a training intervention should boost their performance. At present this remains an open empirical question.

Regardless of how children come to better understand this class of psychogenic phenomena, important changes are clearly taking place between preschool and middle childhood in how children structure the boundaries of these domains. Children appear to go from at first considering these domains to be separate and strictly modular to recognizing that the domains interact with and influence one another. This observation calls into question views in which domain boundaries are considered innately fixed and unchanging (see Gopnik \& Meltzoff, 1997, for discussion of domains and experience). Studies that focus solely on cases in which domain boundaries are appropriate have yielded a picture in which the domains are well established by the preschool years and undergo little structural change. Cross-domain studies such as the present one, however, provide significant test cases of structural invariance in domain reasoning and offer a new perspective on this issue.

\section{ACKNOWLEDGMENTS}

This research was submitted by the first author as a $\mathrm{PhD}$ dissertation at the University of Michigan and was supported by a Student Award Program grant from the Blue Cross and Blue Shield of Michigan Foundation. This research was supported in part by NICHD grant R01-HD36043 to S. Gelman. The authors thank Harold Stevenson, Jennifer Myers, John Hagen, and Scott Paris for their constructive feedback and Alison Gopnik and two anonymous reviewers for commenting on a previous draft. They are grateful to the kindergarten and the second- and fifth-grade students of Hoben and Isbister schools in PlymouthCanton and the teachers and administrative staff there who encouraged the research. Also, many thanks go to the children and staff of Green House Montessori, Little Tots of Plymouth and Livonia, Rainbow Rascals, River Oaks Daycare, and St. Thomas 
Elementary school, who generously supported this work, as well as Farhad Attary, Haslindawati Hassan, Courtney Leshin, Tenisha Mason, and Elisa Rubin for their research assistance.

\section{ADDRESSES AND AFFILIATIONS}

Corresponding author: Susan A. Gelman, Department of Psychology, 525 E. University Ave., University of Michigan, Ann Arbor, MI 48109-1109; e-mail: gelman@umich.edu. Paul Notaro is at University of Missouri, at St. Louis, MO; and Marc A. Zimmerman is at the University of Michigan, at Ann Arbor, MI.

\section{REFERENCES}

Au, T. K., \& Romo, L. F. (1999). Mechanical causality in children's "folkbiology." In D. Medin \& S. Atran (Eds.), Folkbiology. Cambridge, MA: MIT Press.

Au, T. K., Sidle, A. L., \& Rollins, K. B. (1993). Developing an intuitive understanding of conservation and contamination: Invisible particles as a plausible mechanism. Developmental Psychology, 29, 286-299.

Bibace, R., \& Walsh, M. E. (1980). Development of children's concepts of illness. Pediatrics, 66, 912-917.

Burbach, D., \& Peterson, L. (1986). Children's concepts of physical illness: A review and critique of the cognitivedevelopmental literature. Health Psychology, 5, 307-325.

Callanan, M. A., \& Oakes, L. M. (1992). Preschoolers' questions and parents' explanations: Causal thinking in everyday activity. Cognitive Development, 7, 213-233.

Carey, S. (1985). Conceptual change in childhood. Cambridge, MA: MIT Press.

Carey, S. (1995). On the origin of causal understanding. In D. Sperber, D. Premack, \& A. J. Premack (Eds.), Causal cognition (pp. 268-302). Oxford, U.K.: Clarendon Press.

Charman, T., \& Chandiramani, S. (1995). Children's understanding of physical illnesses and psychological states. Psychology and Health, 10, 145-153.

Coley, J. D. (1995). Emerging differentiation of folkbiology and folkpsychology: Attributions of biological and psychological properties to living things. Child Development, $66,1856-1874$.

Estes, D., Wellman, H. M., \& Woolley, J. D. (1989). Children's understanding of mental phenomena. Advances in Child Development and Behavior, 22, 41-87.

Garralda, M. E., \& Bailey, D. (1987). Psychosomatic aspects of children's consultations in primary care. European Archives of Psychiatry and Neurological Sciences, 236, 319322.

Garralda, M. E., \& Bailey, D. (1990). Pediatrician identification of psychological factors associated with general pediatric consultations. Journal of Psychosomatic Research, 34, 303-312.

Gopnik, A., \& Meltzoff, A. N. (1997). Words, thoughts, and theories. Cambridge, MA: MIT Press.

Gopnik, A., \& Wellman, H. M. (1994). The theory theory. In
L. A. Hirschfeld \& S. A. Gelman (Eds.), Mapping the mind: Domain specificity in cognition and culture (pp. 257-293). New York: Cambridge University Press.

Harbeck, C., \& Peterson, L. (1992). Elephants dancing in my head: A developmental approach to children's concepts of specific pains. Child Development, 63, 138-149.

Harris, P. L. (1989). Children and emotion: The development of psychological understanding. New York: Basil Blackwell.

Harris, P. L., Brown, E., Marriot, C., Whithall, S., \& Harmer, S. (1991). Monsters, ghosts, and witches: Testing the limits of the fantasy-reality distinction in young children. British Journal of Developmental Psychology, 9, 105-123.

Hatano, G., \& Inagaki, K. (1994). Young children's naive theory of biology. Cognition, 50, 171-188.

Inagaki, K., \& Hatano, G. (1993). Young children's understanding of the mind-body distinction. Child Development, 64, 1534-1549.

Kalish, C. (1996). Causes and symptoms in preschoolers' conceptions of illness. Child Development, 67, 16471670.

Kalish, C. (1997). Preschoolers' understanding of mental and bodily reactions to contamination: What you don't know can hurt you, but cannot sadden you. Developmental Psychology, 33, 79-91.

Kaplan, H. I. (1980). Treatment of psychosomatic disorders. In H. I. Kaplan, A. M. Freedman, \& B. J. Saddock (Eds.), Comprehensive textbook of psychiatry IV (3rd ed.) (Vol. 2, pp. 1973-1980). New York: Williams \& Wilkins.

Keil, F. C. (1992). The origins of an autonomous biology. In M. R. Gunnar \& M. Maratsos (Eds.), Modularity and constraints in language and cognition (pp. 103-137). Hillsdale, NJ: Erlbaum.

Keil, F. C. (1994). The birth and nurturance of concepts by domains: The origins of concepts of living things. In L. A. Hirschfeld \& S. A. Gelman (Eds.), Mapping the mind: Domain specificity in cognition and culture (pp. 234-254). New York: Cambridge University Press.

Kister, M. C., \& Patterson, C. J. (1980). Children's conceptions of the causes of illness: Understanding of contagion and use of immanent justice. Child Development, 51, 839-846.

Perrin, E. C., \& Gerrity, P. S. (1981). There's a demon in your belly: Children's understanding of illness. Pediatrics, 67, 841-849.

Reister, G., Tress, W., Schepank, H., \& Manz, R. (1989). The epidemiology of psychogenic disorders and consequences for prevention. Psychotherapy and Psychosomatics, 52, 10-20.

Schult, C. A., \& Wellman, H. M. (1997). Explaining human movements and actions: Children's understanding of the limits of psychological explanation. Cognition, 62, 291-324.

Siegal, M. (1988). Children's knowledge of contagion and contamination as causes of illness. Child Development, 59, 1353-1359.

Sigelman, C. K., Estrada, A. L., Derenowski, E. B., \& Woods, T. E. (1996). Intuitive theories of human immunodeficiency virus transmission: Their development 
and implications. Journal of Pediatric Psychology, 21, 555572.

Sigelman, C., Maddock, A., Epstein, J., \& Carpenter, W. (1993). Age differences in understandings of disease causality: AIDS, colds, and cancer. Child Development, 64, 272-284.

Springer, K. (1992). Children's awareness of the biological implications of kinship. Child Development, 63, 950-959.

Springer, K. (1994). Beliefs about illness causality among preschoolers with cancer: Evidence against immanent justice. Journal of Pediatric Psychology, 19, 91-101.

Springer, K. (1996). Young children's understanding of a biological basis for parent-offspring relations. Child Development, 67, 2841-2856.

Springer, K., \& Ruckel, J. (1992). Early beliefs about the cause of illness: Evidence against immanent justice. Cognitive Development, 7, 429-443.

Taplin, J. E., Goodenough, B., Webb, J. R., \& Vogl, L. (1999). Children and pain. In M. Siegal \& C. C. Petersen (Eds.), Children's understanding of biology and health. New York: Cambridge University Press.

Wellman, H. M. (1990). The child's theory of mind. Cambridge, MA: MIT Press.

Wellman, H. M., \& Estes, D. (1986). Early understanding of mental entities: A reexamination of childhood realism. Child Development, 57, 910-923.

Wellman, H. M., \& Gelman, S. A. (1992). Cognitive development: Foundational theories of core domains. Annual Review of Psychology, 43, 337-375. 